\title{
Flutuação de Diptera em granja avícola, Pelotas, Rio Grande do Sul, Brasil ${ }^{1}$
}

\author{
Carla de Lima Bicho²; Lúcia Massutti de Almeida ${ }^{3}$; Paulo Bretanha Ribeiro ${ }^{4}$ \& Paulo Silveira Júnior ${ }^{5}$ \\ 1. Contribuição ${ }^{\circ}$ 1448, Depto de Zoologia, Universidade Federal do Paraná (UFPR). Parte da Tese de Doutorado da autora \\ sênior, Curso de Pós-Graduação em Ciências Biológicas, Área de Concentração em Entomologia, UFPR. \\ 2. Escola de Educação, Universidade Católica de Pelotas, Caixa Postal 402, 96010-000, Pelotas, RS, Brasil. \\ (clbicho@phoenix.ucpel.tche.br) \\ 3. Depto de Zoologia, Setor de Ciências Biológicas, UFPR, Caixa Postal 19020, 81531-980, Curitiba, PR, Brasil. (lalmeida@ufpr.br) \\ 4. Depto de Microbiologia e Parasitologia, Instituto de Biologia, Universidade Federal de Pelotas (UFPel), Caixa Postal 354, \\ 96010-900, Pelotas, RS, Brasil. (bretanha@ufpel.tche.br) \\ 5. Depto de Matemática, Estatística e Computação, Instituto de Física e Matemática, UFPel, Caixa Postal 354, 96010-900, Pelotas, \\ RS, Brasil. (prpd@ufpel.tche.br)
}

\begin{abstract}
Fluctuation of Diptera in poultry house, Pelotas, Rio Grande do Sul, Brazil. In order to investigate the population fluctuation of Diptera in a poultry house in Pelotas, Rio Grande do Sul, Brazil, six collection methods were utilized: 1 ( 0 to 7 day-old feces from chickens), 2 ( 7 to 14 day-old feces), 3 (14 to 21 dayold feces), 4 ( 0 to 21 day-old feces), 5 (accumulated feces) and 6 (tube trap). Analyses of polynomial regression were accomplished independent of the collection method. The survey was conducted from August 1998 to July 1999 in chicken houses at the Conjunto Agrotécnico Visconde da Graça. A total of 28,720 Diptera were collected, including the following species: Coproica sp. and Telomerina flavipes (Meigen, 1830) (15,640); Drosophila repleta Wollaston, 1858 (9,229); Dohrniphora cornuta (Bigot, 1857) (2,539); Ischiolepta scabricula (Haliday, 1833) (544); Lestodiplosis sp. (320); Muscina stabulans (Fallen, 1817) (159); Musca domestica L., 1758 (143); Drosophila melanogaster Meigen, 1830 (95); Telmatoscopus albipunctatus Williston, 1893 (21); Rhegmoclema sp. (14); Fannia canicularis (L., 1761) (7); Stomoxys calcitrans (L., 1758) (2); and unidentified species of Psychodidae (6) and Muscidae (1). The greatest number of species occurred in October, November and December and the fewest in August, September and April. The greatest abundance of Diptera was recorded in October $(9,092)$, while the lowest index of capture was noted in April (658). The population fluctuation was estimated for Coproica sp. and T. flavipes, D. repleta, D. cornuta, I. scabricula and Lestodiplosis sp.
\end{abstract}

KEYWORDS. Diptera, fluctuation, poultry house.

\section{INTRODUÇÃO}

Em locais de confinamento de animais domésticos, especialmente em granjas avícolas, existe uma produção significativa de esterco, que funciona como substrato para o desenvolvimento de inúmeras espécies de artrópodes, principalmente de dípteros sinantrópicos. Estes, muitas vezes, são considerados pragas devido não somente à sua densidade, mas, principalmente, à tolerância biológica de seus hospedeiros (WALLNER, 1987). Populações de dípteros encontradas nos aviários, quando ultrapassam o nível de dano econômico, ocasionam alguns problemas devido ao hábito que possuem de defecar e regurgitar sobre as superfícies. Deixam manchas nos equipamentos da granja, o que, nas instalações luminosas, causam uma redução no nível de iluminação. Podem ainda veicular patógenos, principalmente, quando pousam em ovos recémcolocados (Axtell, 1985; Axtell \& Arends, 1990). Através do seu corpo, trato digestivo ou até mesmo das suas fezes, as moscas podem ser transmissoras de diversos microorganismos tanto para o homem como para as aves (Harwood \& JAMEs, 1979; Axtell, 1985; Axtell \& ARENDS, 1990; MARICONI et al., 1999).

No Brasil, os estudos sobre a diversidade de dípteros encontrados em aviários foram realizados somente na Região Sudeste (BRuno et al., 1993; LOMÔNACO \& Prado, 1994; Fernandes et al., 1995 e Avancini \&
Silveira, 2000). Dentre os dípteros sinantrópicos, destaca-se a espécie cosmopolita Musca domestica L., 1758 (Muscidae) que, devido à freqüência, abundância e importância na saúde pública (PFeIFFer \& Axtell, 1980; Axtell, 1986a; Axtell \& Arends, 1990; Bruno et al., 1993; LomônaCo \& Prado, 1994; Fernandes et al., 1995), vem sendo alvo de programas de manejo integrado de pragas (Axtell, 1986b; Axtell \& ARends, 1990; Axtell \& STINNER, 1990; WATSON et al., 1994).

Objetivou-se identificar os dípteros que ocorrem em uma granja avícola em Pelotas, Rio Grande do Sul, bem como estimar a flutuação populacional das espécies mais freqüentes.

\section{MATERIAL E MÉTODOS}

O trabalho foi conduzido na granja do Conjunto Agrotécnico Visconde da Graça (CAVG), pertencente à Universidade Federal de Pelotas, Pelotas $\left(31^{\circ} 34^{\prime}\right.$ 'S; $\left.52^{\circ} 23^{\prime} \mathrm{W}\right), \mathrm{RS}$, no período de agosto de 1998 a julho de 1999. A granja, distante $7 \mathrm{~km}$ do centro da cidade, tem capacidade para alojar 14000 aves. As instalações abrangem uma área total de um hectare, onde estão distribuídos nove galpões galvanizados fechados, sendo três para a criação de galinhas poedeiras ou matrizes em cama com maravalhas e seis para criação de poedeiras em gaiola. Cada galpão apresenta área de $245 \mathrm{~m}^{2}(20 \mathrm{~m}$ de comprimento x 12,25 m de largura) e capacidade para 
abrigar de 1200 a 3400 aves, dependendo do tipo de criação. Nas proximidades da granja, há estábulo para bovinos de leite e criação de suínos e coelhos, além do plantio de milho, pastagens e horticultura.

As coletas foram realizadas no galpão de poedeiras em cama com maravalhas, que apresentava um puleiro central ( $14 \mathrm{~m}$ de comprimento $\times 10 \mathrm{~m}$ de largura $\times 0,6 \mathrm{~m}$ de altura) formado por muretas de concreto e coberto por um estrado de madeira com tela, no qual eram suspensos os bebedouros e os comedouros. No interior do puleiro, as fezes das aves ficavam retidas durante todo o período de permanência do lote, funcionando desta forma como área de refúgio e alimentação de diversas espécies de artrópodes. As aves não tinham acesso às fezes. A área de circulação ao redor do puleiro apresentava uma camada de maravalhas com cerca de $10 \mathrm{~cm}$ de altura, reposta sempre que necessário. O lote contendo 1075 galinhas da linhagem "Isa Brown" foi instalado neste galpão em 16.I.1998, com 90 dias de idade, tendo sido descartado em 20.X.1999. Durante todo o período experimental não foi aplicado nenhum produto químico para controle de artrópodes.

Para a coleta dos dípteros foram utilizados seis métodos. Nos métodos de $1 \mathrm{a} 4$, as bandejas plásticas (20 $\mathrm{cm} \times 20 \mathrm{~cm} \times 10 \mathrm{~cm}$ ), com tampas, eram preenchidas com aproximadamente um quilo de fezes de galinhas (com menos de $24 \mathrm{~h}$ ), com três repetições para cada método. As bandejas dos métodos 1 e 4 com fezes recentes eram expostas imediatamente no galpão de poedeiras em cama, enquanto que as bandejas dos métodos 2 e 3 eram isoladas em gaiola, com tela contra insetos, até o momento da exposição, que correspondeu a 0-7 dias e 7-14 dias, respectivamente. As bandejas do método 4 ficavam expostas durante três semanas, as dos métodos 1,2 e 3, apenas uma semana cada. Devido ao tipo de estrutura deste galpão, as fezes utilizadas nos métodos 1 a 4 foram recolhidas no galpão de poedeiras em gaiolas.

As bandejas com fezes, ao findar o período de exposição no galpão, eram levadas ao laboratório, colocadas dentro dos funis de alumínio $(\phi=35 \mathrm{~cm})$, cobertos com toucas de pano e com frascos plásticos transparentes acoplados (coletores com $200 \mathrm{ml}$ de capacidade) contendo álcool $70 \%$, por sete dias, em câmara climatizada a $\pm 25^{\circ} \mathrm{C}$, U.R. $\geq 80 \%$, para a captura dos dípteros. Terminado este período, os frascos coletores eram substituídos e lâmpadas ( $40 \mathrm{~W}$ ) eram colocadas incidindo sobre cada funil, por mais quatro dias.

O método 5 consistiu na coleta de três amostras com cerca de um quilo de fezes acumuladas sob o estrado do galpão (de poedeiras), posteriormente acondicionadas em bandejas. No laboratório, as etapas cumpridas para obtenção dos artrópodes foram as mesmas executadas para os métodos 1 a 4. Em complementação, também foi utilizado o método da flotação (ToBin \& PitTs, 1999), colocando-se pequenas quantidades de fezes em uma bandeja plástica branca $(29 \mathrm{~cm} \times 34 \mathrm{~cm} \times 5 \mathrm{~cm})$ contendo água, para remoção dos dípteros. Previamente, as amostras eram colocadas no congelador, onde permaneciam por 24 horas para sacrificar os artrópodes.

No método 6 foi utilizada armadilha conhecida como "tube trap", idealizada por J. J. Arends e descrita por
SAFRIT \& AXTEll (1984). Esta armadilha consiste de um tubo de PVC com $4 \mathrm{~cm}$ de diâmetro e $17 \mathrm{~cm}$ de comprimento, dentro do qual se insere um papelão corrugado, com $17 \mathrm{~cm}$ de largura por $25 \mathrm{~cm}$ de comprimento, enrolado em espiral (de forma que as ondulações fiquem no sentido longitudinal do tubo). Oito armadilhas foram dispostas pelo chão do galpão, junto à mureta de concreto, fixadas com tijolos e cobertas com maravalhas para evitar o movimento das mesmas pelo esgravatar das galinhas. Para o monitoramento dos artrópodes foram realizadas duas visitas por mês: uma até o dia dez e a outra após o dia vinte. A cada visita, as armadilhas eram recolhidas e examinadas separadamente, retirando-se o papelão e removendo os artrópodes para posterior identificação e contagem. Após este procedimento, a artropodofauna era devolvida à cama $\mathrm{e}$ as armadilhas recolocadas em seus respectivos locais.

No momento da triagem, as espécies diminutas Coproica sp. e Telomerina flavipes (Meigen, 1830) (Sphaeroceridae) foram agrupadas como sendo uma mesma morfoespécie e por isso estão sendo analisadas em conjunto neste trabalho. Porém, identificação posteriormente feita por um especialista indicou a ocorrência de duas espécies distintas neste agrupamento. Para a identificação dos dípteros, foram utilizados os trabalhos de Axtell (1986a), Colless \& McAlpine (1991), CURRAn (1965) e MARICONI et al. (1999).

A flutuação populacional foi estimada tomando-se as observações, referentes aos seis métodos de coleta, previamente transformadas em $\sqrt{x+0,5}$, como repetição e utilizando-se o método da análise de regressão polinomial.

As espécies estudadas encontram-se depositadas na coleção entomológica "Pe. Jesus Santiago Moure", Departamento de Zoologia, Universidade Federal do Paraná, Curitiba.

\section{RESULTADOS E DISCUSSÃO}

Durante o período de estudo, foram capturados 46715 artrópodes, dos quais $28720(61,47 \%)$ correspondem a dípteros: Coproica sp.; Telomerina flavipes; Drosophila repleta Wollaston, 1858; Dohrniphora cornuta (Bigot, 1857); Ischiolepta scabricula (Haliday, 1833); Lestodiplosis sp.; Muscina stabulans (Fallen, 1817); Musca domestica; Drosophila melanogaster Meigen, 1830; Telmatoscopus albipunctatus Williston, 1893; Rhegmoclema sp.; Fannia canicularis (L., 1761); Stomoxys calcitrans (L., 1758); uma espécie não identificada de Psychodidae e uma de Muscidae (tab. I).

Ao longo dos doze meses de coleta, houve uma variação mensal no número de espécies capturadas, tendo ocorrido o maior número de espécies (9) nos meses de outubro a dezembro, e o menor número (5), em agosto, setembro e abril (tab. II), sendo que agosto e setembro apresentaram temperaturas médias mensais muito baixas $\left(13^{\circ} \mathrm{C} \mathrm{e} 14^{\circ} \mathrm{C}\right.$, respectivamente) (fig. 1$)$.

Dípteros foram observados ao longo de todo o ano, porém em outubro registrou-se o maior número de indivíduos (9092) (fig. 2, tab. II), o que coincide com um súbito aumento da temperatura média mensal de $14^{\circ} \mathrm{C}$ 
Tabela I. Freqüência de captura das espécies de Diptera em galpão de aves poedeiras, no período de agosto de 1998 a julho de 1999, em Pelotas, RS (Abs, absoluta; Rel, relativa).

\begin{tabular}{lrr}
\hline \multirow{2}{*}{ Espécies } & \multicolumn{2}{c}{ Freqüência } \\
\cline { 2 - 3 } & Abs $\left(\mathrm{n}^{\circ}\right)$ & \multicolumn{1}{c}{ Rel $(\%)$} \\
\hline Coproica sp. e Telomerina flavipes & 15640 & 33,480 \\
Drosophila repleta & 9229 & 19,756 \\
Dohrniphora cornuta & 2539 & 5,435 \\
Ischiolepta scabricula & 544 & 1,165 \\
Lestodiplosis sp. & 320 & 0,685 \\
Muscina stabulans & 159 & 0,340 \\
Musca domestica & 143 & 0,306 \\
Drosophila melanogaster & 95 & 0,203 \\
Telmatoscopus albipunctatus & 21 & 0,045 \\
Rhegmoclema sp. & 14 & 0,030 \\
Fannia canicularis & 7 & 0,015 \\
Stomoxys calcitrans & 2 & 0,004 \\
Psychodidae sp. 1 & 6 & 0,013 \\
Muscidae sp. 1 & 1 & 0,002 \\
Outros insetos & 13693 & 29,312 \\
Outros artrópodes & 4302 & 9,209 \\
\hline Total & 46715 & 100,000 \\
\hline
\end{tabular}

para $20,7^{\circ} \mathrm{C}$ (fig. 1). Julho foi o segundo mês de maior coleta (7618) (tab. II). O menor índice de captura foi assinalado em abril (658) (tab. II), quando a temperatura média mensal, depois de seis meses permanecendo acima dos $20,5^{\circ} \mathrm{C}$, teve brusca diminuição para $16,8^{\circ} \mathrm{C}$ (fig. 1 ). A maior frequiência de dípteros foi registrada nos intervalos de temperatura média mensal entre $12,4^{\circ} \mathrm{C}$ a $15,2^{\circ} \mathrm{C}$ $(37,17 \%)$ e $18,0^{\circ} \mathrm{C}$ a $20,8^{\circ} \mathrm{C}(35,53 \%)$ (tab. III). Pela análise de regressão, o modelo cúbico y $=-0,352+36,186 \mathrm{X}$ $7,395 X^{2}-0,413 X^{3}$ foi significativo $(7,8 \%)$, com coeficiente de determinação de $47,6 \%$.

Coproica sp. e Telomerina flavipes foram as espécies mais abundantes, com 15640 espécimens coletados, representando $54,47 \%$ da ordem (tab. II). No período de amostragem, foram capturadas em 11 dos 12 meses, não havendo captura em abril (fig. 3), provavelmente em função da redução de temperatura de $23,3^{\circ} \mathrm{C}$ para $16,8^{\circ} \mathrm{C}$ (fig. 1 ). $\mathrm{O}$ acme populacional destas espécies aconteceu em outubro, quando foram coletados 8098 espécimens (tab. II), o que coincide com um súbito aumento da temperatura média mensal de $14^{\circ} \mathrm{C}$ para $20,7^{\circ} \mathrm{C}$ (fig. 1). Em julho registrou-se também alto número destes insetos (6518). A menor captura foi em fevereiro (5) (tab. II). Estas espécies ocorreram em maior frequiência, $45,31 \%$ e $51,81 \%$, respectivamente, nas faixas de temperatura de $12,4^{\circ} \mathrm{C}$ a $15,2^{\circ} \mathrm{C}$ e $18,0^{\circ} \mathrm{C}$ a $20,8^{\circ} \mathrm{C}$ (tab. III). O modelo que melhor se ajustou aos dados foi o cúbico, $\mathrm{y}=-18,840+$ $44,586 X-10,042 X^{2}+0,580 X^{3}$, significativo a $3,6 \%$, com coeficiente de determinação de $58,6 \%$.

Drosophila repleta esteve presente durante todo o ano, apresentando acme populacional em maio (1695) (fig. 4). Nos meses de junho, novembro, fevereiro e julho observou-se alto índice de ocorrência, com 1396, 1072, 1067 e 1067 espécimens coletados, respectivamente. A menor captura foi assinalada em março (136) (tab. II). O intervalo de temperatura média mensal em que se observou maior frequiência $(32,45 \%)$ foi o de $12,4^{\circ} \mathrm{C}$ a $15,2^{\circ} \mathrm{C}$ (tab. III). O modelo ajustado foi linear, $\mathrm{y}=17,117+1,405 \mathrm{X}$, significativo a $5,8 \%$, porém com coeficiente de determinação muito baixo $(29,2 \%)$.

Fernandes et al. (1995), em levantamento dos artrópodes que ocorreram em esterco acumulado em granja de galinhas poedeiras, em Uberlândia, MG, registraram um alto índice de captura de uma espécie de Drosophilidae não identificada, ou seja, 18823 dos 28242 dípteros coletados em 10 dos 11 meses de experimento, com picos populacionais em fevereiro, março e agosto, não havendo captura em abril.

Tabela II. Espécies de Diptera e número total de indivíduos capturados em galpão de aves poedeiras, no período de agosto de 1998 a julho de 1999, em Pelotas, RS.

\begin{tabular}{|c|c|c|c|c|c|c|c|c|c|c|c|c|c|}
\hline \multirow[b]{2}{*}{ Famílias/Espécies } & \multicolumn{5}{|c|}{1998} & \multicolumn{7}{|c|}{1999} & \multirow[b]{2}{*}{ Total } \\
\hline & Ago & Set & Out & Nov & Dez & Jan & Fev & Mar & Abr & Maio & Jun & Jul & \\
\hline \multicolumn{14}{|l|}{ Psychodidae } \\
\hline Telmatoscopus albipunctatus & & & & 6 & 9 & 4 & 1 & & & & 1 & & 21 \\
\hline Espécie não identificada & & & 6 & & & & & & & & & & 6 \\
\hline \multicolumn{14}{|l|}{ Scatopsidae } \\
\hline Rhegmoclema sp. & 1 & 5 & 5 & 1 & & & & & 1 & & & 1 & 14 \\
\hline \multicolumn{14}{|l|}{ Cecidomyiidae } \\
\hline Lestodiplosis sp. & 41 & 25 & 189 & 11 & 28 & 12 & 1 & 9 & 1 & & 2 & 1 & 320 \\
\hline \multicolumn{14}{|l|}{ Phoridae } \\
\hline Dohrniphora cornuta & 427 & 22 & 273 & 37 & 21 & 614 & 19 & 1060 & 58 & 5 & 1 & 2 & 2539 \\
\hline \multicolumn{14}{|l|}{ Sphaeroceridae } \\
\hline Coproica sp. e Telomerina flavipes & 194 & 313 & 8098 & 331 & 43 & 16 & 5 & 20 & & 40 & 62 & 6518 & 15640 \\
\hline Ischiolepta scabricula & & & 1 & 1 & 1 & 13 & 17 & 195 & 215 & 39 & 36 & 26 & 544 \\
\hline \multicolumn{14}{|l|}{ Drosophilidae } \\
\hline Drosophila repleta & 189 & 343 & 502 & 1072 & 798 & 581 & 1067 & 136 & 383 & 1695 & 1396 & 1067 & 9229 \\
\hline D. melanogaster & & & 17 & 71 & 3 & & 1 & & & & 1 & 2 & 95 \\
\hline \multicolumn{14}{|l|}{ Faniidae } \\
\hline Fannia canicularis & & & & & & 7 & & & & & & & 7 \\
\hline \multicolumn{14}{|l|}{ Muscidae } \\
\hline Musca domestica & & & & & 54 & 87 & 1 & 1 & & & & & 143 \\
\hline Muscina stabulans & & & & 1 & 1 & & & & & 157 & & & 159 \\
\hline Stomoxys calcitrans & & & & & & & & & & 1 & & 1 & 2 \\
\hline Espécie não identificada & & & 1 & & & & & & & & & & 1 \\
\hline Número de espécies & 5 & 5 & 9 & 9 & 9 & 8 & 8 & 6 & 5 & 6 & 7 & 8 & \\
\hline Número de indivíduos & 852 & 708 & 9092 & 1531 & 958 & 1334 & 1112 & 1421 & 658 & 1937 & 1499 & 7618 & 28720 \\
\hline
\end{tabular}




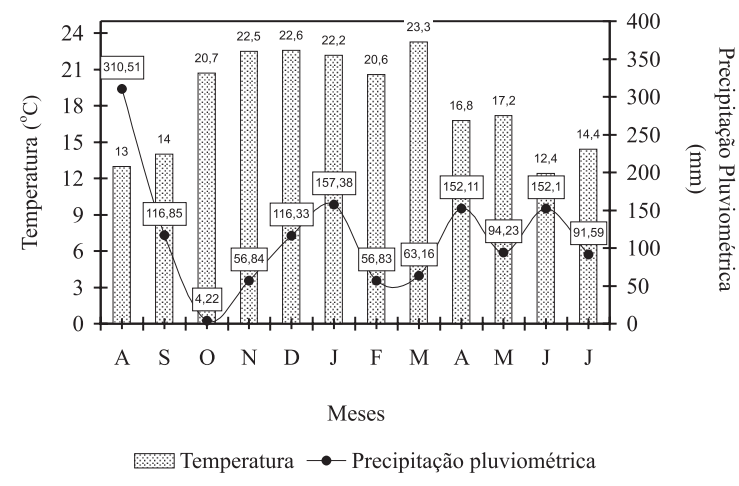

Fig. 1. Temperatura média mensal e precipitação pluviométrica, no período de agosto de 1998 a julho de 1999, na Estação Climatológica de Conjunto Agrotécnico Visconde da Graça, Pelotas, RS.

Coproica sp., Telomerina flavipes e Drosophila repleta foram abundantes, provavelmente por serem saprófagas e dependerem diretamente do substrato presente na granja. Segundo Ricklefs (1996), os indivíduos são mais numerosos onde os recursos por eles utilizados são mais abundantes, o que define a capacidade suporte do ambiente.

Dohrniphora cornuta ocorreu em todos os meses amostrados, sendo que o seu pico populacional foi registrado em março (1060) (fig. 5; tab. II), que apresentou a temperatura média mensal mais alta $\left(23,3^{\circ} \mathrm{C}\right)$ (fig. 1$)$. Os meses de janeiro, agosto e outubro também obtiveram consideráveis índices de captura, 614, 427 e 237, respectivamente, quando comparados com os demais (tab. II). A menor ocorrência desta espécie aconteceu em junho (1) (tab. II), com temperatura média mensal mais baixa $\left(12,4^{\circ} \mathrm{C}\right)$ (fig. 1). A grande maioria dos espécimens de $D$. cornuta $(68,22 \%)$ foi coletada num período com temperatura média mensal entre $20,8^{\circ} \mathrm{C}$ a $23,3^{\circ} \mathrm{C}$ (tab. III). A análise de regressão polinomial realizada não identificou modelo significativo para a flutuação populacional desta espécie.

Ischiolepta scabricula foi capturada nos meses de outubro a julho, com o acme populacional em abril (215) (fig. 6). Os menores índices foram registrados de outubro a dezembro (1) (tab. II). Apresentou os maiores índices de captura nas faixas de temperatura de $15,2^{\circ} \mathrm{C}$ a $18,0^{\circ} \mathrm{C}$ $(46,69 \%)$ e de $20,8^{\circ} \mathrm{C}$ a $23,3^{\circ} \mathrm{C}(38,60 \%)$ (tab. III). O modelo que melhor se ajustou aos dados foi o cúbico, $\mathrm{y}=5,673$ $5,206 X+1,287 X^{2}-0,072 X^{3}$, significativo a $3,3 \%$, com

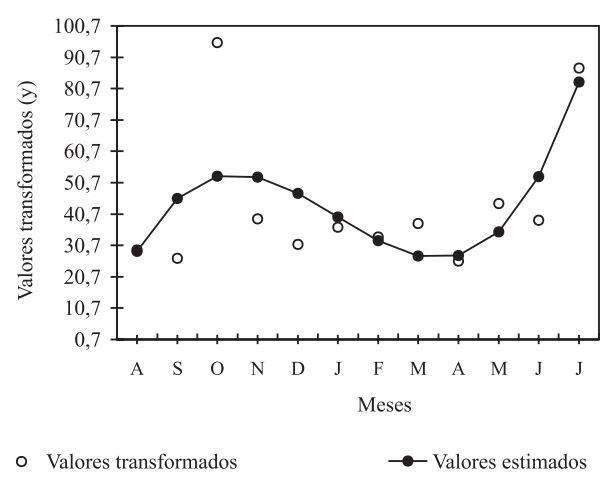

Fig. 2. Flutuação populacional das espécies de Diptera em granja avícola, no período de agosto de 1998 a julho de 1999, Pelotas, RS.

coeficiente de determinação de $69,3 \%$.

Lestodiplosis sp. apresentou um acme populacional em outubro (189) (fig. 7). O menor índice de coleta foi em fevereiro, abril e julho (1). Em maio não houve captura deste díptero (tab. II). Ocorreu em grande quantidade quando a temperatura média mensal esteve entre $18,0^{\circ} \mathrm{C}$ a $20,8^{\circ} \mathrm{C}(59,38 \%)$ (tab. III). O modelo ajustado foi o linear, $y=8,416-0,698 X$, significativo a $3,4 \%$, com coeficiente de determinação de $48,2 \%$.

Segundo Odum (1988), as flutuações anuais podem ser controladas por fatores extrínsecos, como temperatura e precipitação, e por fatores intrínsecos, como disponibilidade de alimento e inimigos naturais. Estes fatores explicam as variações específicas que ocorreram nas populações estudadas, as quais apresentaram diferenças nos modelos matemáticos expressos e também na densidade populacional, demonstrando que as exigências abióticas e bióticas são próprias de cada espécie.

As espécies de dípteros comentadas a seguir, devido às baixas densidades populacionais e freqüências mensais, não tiveram suas flutuações populacionais estimadas. Foram capturados 159 espécimens de Muscina stabulans nos meses de novembro, dezembro e maio, sendo maio o de maior captura (157) (tab. II). Estes foram os meses em que as temperaturas médias mensais estiveram mais altas, $22,5^{\circ} \mathrm{C}, 22,6^{\circ} \mathrm{C}$ e $23,3^{\circ} \mathrm{C}$, respectivamente (fig. 1). A ocorrência de $M$. stabulans foi assinalada por BRUNo et al. (1993), em levantamento de moscas sinantrópicas e seus predadores encontrados

Tabela III. Distribuição de frequiências da captura das espécies de Diptera, de acordo com a temperatura média mensal, no Conjunto Agrotécnico Visconde da Graça, no período de agosto de 1998 a julho de 1999, em Pelotas, RS (Abs, absoluta; Rel, relativa; $\Sigma$, somatório das espécies de dípteros capturadas).

\begin{tabular}{|c|c|c|c|c|c|c|c|c|c|c|c|c|}
\hline \multirow[b]{2}{*}{$\begin{array}{c}\text { Temperatura } \\
\left({ }^{\circ} \mathrm{C}\right)\end{array}$} & \multicolumn{2}{|c|}{$\begin{array}{c}\text { Coproica sp. e } \\
\text { Telomerina flavipes }\end{array}$} & \multicolumn{2}{|c|}{$\begin{array}{l}\text { Drosophila } \\
\text { repleta }\end{array}$} & \multicolumn{2}{|c|}{$\begin{array}{l}\text { Dohrniphora } \\
\text { cornuta }\end{array}$} & \multicolumn{2}{|c|}{$\begin{array}{r}\text { Ischiolepta } \\
\text { scabricula }\end{array}$} & \multicolumn{2}{|c|}{ Lestodiplosis sp. } & \multicolumn{2}{|c|}{$\Sigma$} \\
\hline & $\begin{array}{l}\text { Abs } \\
\left(\mathrm{n}^{\circ}\right)\end{array}$ & $\begin{array}{l}\text { Rel } \\
(\%)\end{array}$ & $\begin{array}{l}\text { Abs } \\
\left(\mathrm{n}^{\circ}\right)\end{array}$ & $\begin{array}{l}\text { Rel } \\
(\%)\end{array}$ & $\begin{array}{l}\text { Abs } \\
\left(n^{\circ}\right)\end{array}$ & $\begin{array}{l}\text { Rel } \\
(\%)\end{array}$ & $\begin{array}{l}\text { Abs } \\
\left(\mathrm{n}^{\circ}\right)\end{array}$ & $\begin{array}{l}\text { Rel } \\
(\%)\end{array}$ & $\begin{array}{l}\text { Abs } \\
\left(n^{\circ}\right)\end{array}$ & $\begin{array}{l}\text { Rel } \\
(\%)\end{array}$ & $\begin{array}{l}\text { Abs } \\
\left(\mathrm{n}^{\circ}\right)\end{array}$ & $\begin{array}{l}\text { Rel } \\
(\%)\end{array}$ \\
\hline $12,4 \mid-15,2$ & 7087 & 45,31 & 2995 & 32,45 & 452 & 17,80 & 62 & 11,40 & 69 & 21,56 & 10677 & 37,17 \\
\hline $15,2 \mid-18,0$ & 40 & 0,26 & 2078 & 22,52 & 63 & 2,48 & 254 & 46,69 & 1 & 0,31 & 2595 & 9,04 \\
\hline $18,0 \mid-20,8$ & 8103 & 51,81 & 1569 & 17,00 & 292 & 11,50 & 18 & 3,31 & 190 & 59,38 & 10204 & 35,53 \\
\hline $20,8|-| 23,3$ & 410 & 2,62 & 2587 & 28,03 & 1732 & 68,22 & 210 & 38,60 & 60 & 18,75 & 5244 & 18,26 \\
\hline Total & 15640 & 100,00 & 9229 & 100,00 & 2539 & 100,00 & 544 & 100,00 & 320 & 100,00 & 28720 & 100,00 \\
\hline
\end{tabular}



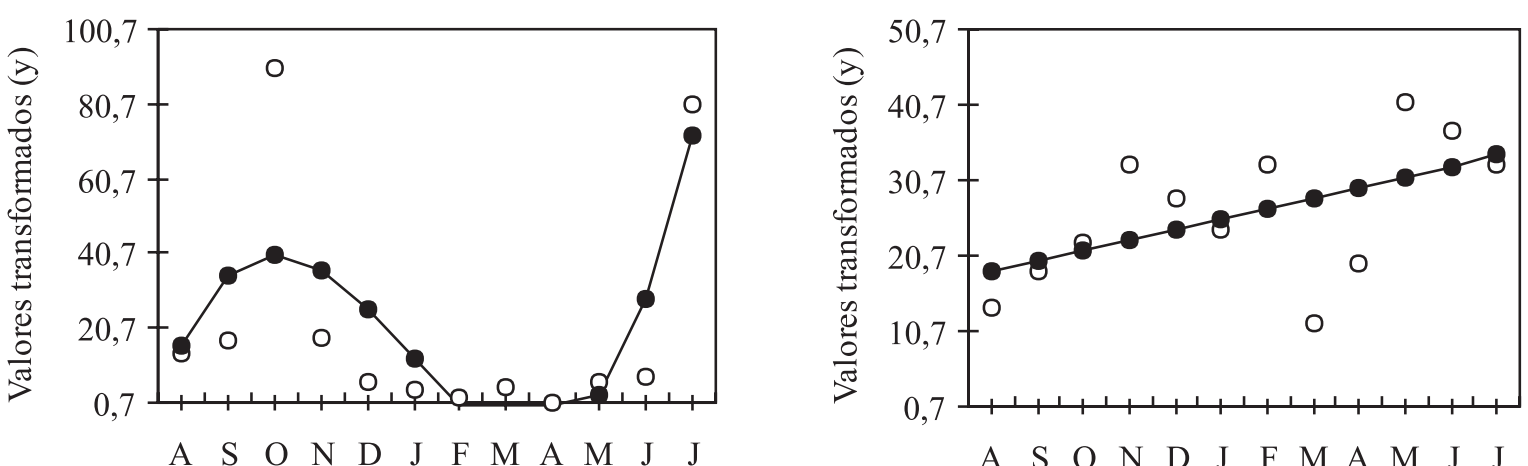

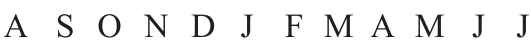

3

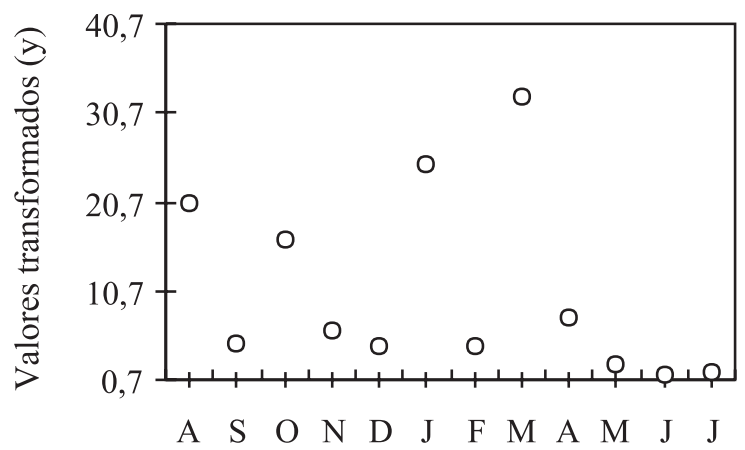

5
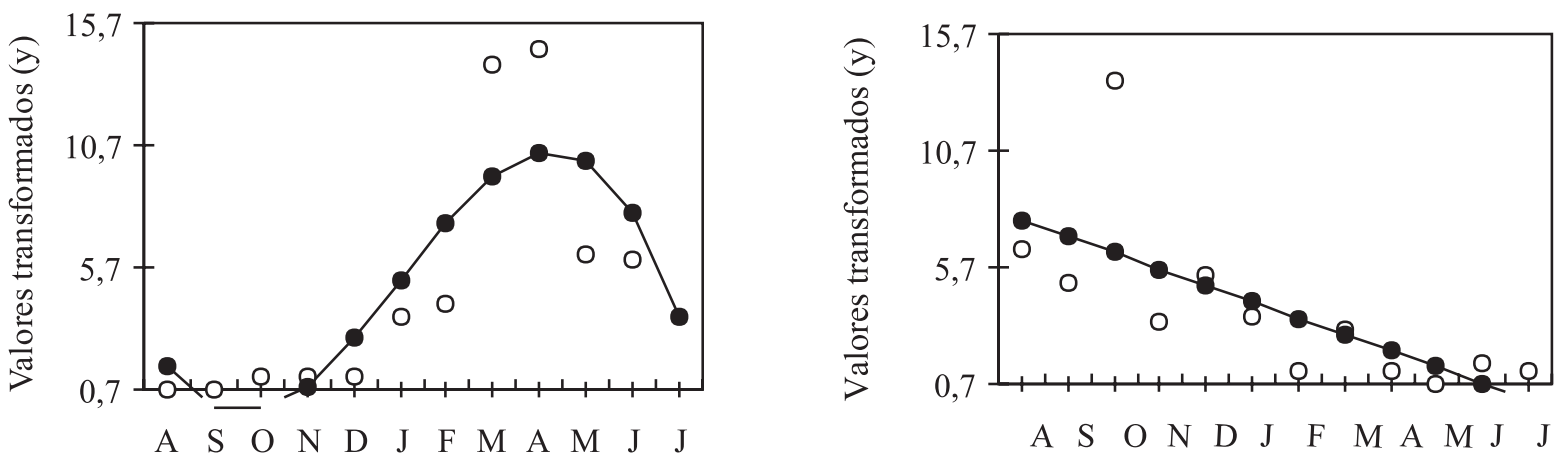

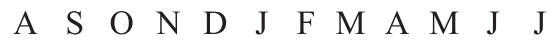

6

Figs. 3-7. Flutuação populacional das espécies de Diptera em granja avícola, no período de agosto de 1998 a julho de 1999, Pelotas, RS: 3 , Coproica sp. e Telomerina flavipes (Sphaeroceridae); 4, Drosophila repleta (Drosophilidae); 5, Dohrniphora cornuta (Phoridae); 6 , Ischiolepta scabricula (Sphaeroceridae); 7, Lestodiplosis sp. (Cecidomyiidae) (círculo branco, valores transformados; círculo preto, valores estimados).

em esterco de aves poedeiras, em diversos municípios do Estado de São Paulo, pela captura de 117 larvas num montante de 5413 de dípteros sinantrópicos, com predominância em esterco com umidade entre 55 a $75 \%$. Avancini \& Silveira (2000) informaram que foi a segunda espécie mais capturada $(14,92 \%)$ dentre os dípteros muscóideos encontrados em aviário em Monte Mor, SP, sendo superada somente pela Musca domestica.

Um total de 143 espécimens de Musca domestica foi capturado nos meses de dezembro (54), janeiro (87), fevereiro (1) e março (1) (tab. II), cujas temperaturas médias mensais foram de $22,6^{\circ} \mathrm{C}, 22,2^{\circ} \mathrm{C}, 20,6^{\circ} \mathrm{C}$ e $23,3^{\circ} \mathrm{C}$, respectivamente (fig. 1). A espécie foi assinalada como sendo o díptero mais abundante nos levantamentos em granjas avícolas feitos por LOMÔNACO \& PRADO (1994) em Uberlândia, MG (91,82\%) e Avancini \& Silveira (2000) em Monte Mor, SP (83,16\% dos muscóideos capturados). BRUNo et al. (1993), em São Paulo, registraram M. domestica como sendo o segundo díptero mais abundante (30,11\% de larvas) e FernANDEs et al. (1995), estudando os artrópodes que ocorrem em esterco acumulado em granja de galinhas poedeiras em Uberlândia, MG, destacam a terceira colocação para essa espécie $(11,51 \%)$. 
Drosophila melanogaster foi assinalada nos meses de outubro (17), novembro (71), dezembro (3), fevereiro (1), junho (1) e julho (2) (tab. II). Foram capturados 21 espécimens de Telmatoscopus albipunctatus de novembro a fevereiro e junho, sendo a maior ocorrência assinalada em dezembro (9) (tab. II). Espécimens de Rhegmoclema sp. foram capturados de agosto a novembro, abril e julho, com a maior freqüência em setembro e outubro (5) (tab. II).

Fannia canicularis ocorreu somente no mês de janeiro, com a captura de 7 espécimens (tab. II). Foi encontrada por BRUNo et al. (1993), em municípios de São Paulo, juntamente com F. trimaculata (Stein, 1898) e uma espécie do grupo $F$. pusio, sendo que o maior número de larvas de dípteros sinantrópicos pertenciam a $F$. trimaculata, ou seja, 2297 das 5413 larvas capturadas. LOMÔNACO \& PRAdo (1994), ao estudarem a estrutura comunitária e dinâmica populacional de dípteros e seus inimigos naturais em granja avícola em Uberlândia, MG, encontraram poucos faniídeos, estes pertencentes a $F$. trimaculata (14) e F. pusio (Wiedemann, 1830) (3).

Stomoxys calcitrans foi coletada em duas oportunidades, uma em maio (1) e outra em julho (1) (tab. II). BRUNO et al. (1993), registram a ocorrência de larvas de $S$. calcitrans $(1,19 \%$ e $3,95 \%$ do total de larvas de dípteros capturados, respectivamente).

Agradecimentos. Ao CNPq, pela concessão da bolsa; ao Depto de Microbiologia e Parasitologia e ao Conjunto Agrotécnico Visconde da Graça (UFPel), por permitirem a realização deste trabalho em suas instalações; ao médico veterinário Paulo Renato Peixoto Costa (UFPel) (in memoriam), por sua relevante colaboração nas saídas de campo e aos especialistas Dra. Eunice A. Bianchi Galati (USP) (Psychodidae), Dra. Valéria Cid Maia (MNRJ) (Cecidomyiidae), Dr. R. Henry L. Disney (University of Cambridge) (Phoridae), Dr. Carlos Ribeiro Vilela (USP) (Drosophilidae), Dr. Claudio José Barros de Carvalho (UFPR) (Fanniidae) e Dr. Steve Marshall (University of Guelph) (Sphaeroceridae), pelo valioso auxílio na identificação do material.

\section{REFERÊNCIAS BIBLIOGRÁFICAS}

Avancini, R. M. P. \& Silveira, G. A. R. 2000. Age structure and abundance in populations of muscoid flies from a poultry facility in southeast Brasil. Memórias do Instituto Oswaldo Cruz, Rio de Janeiro, 95(2):259-264.

Axtell, R. C. 1985. Arthropod pests of poultry. In: Williams, R. E.; Hall, R. D. et al. eds. Livestock Entomology. New York, Willey Interscience. p.269-295.

_- . 1986a. Fly control in confined livestock and poultry production. Technical Monograph, Greensboro, CIBAGEIGY. 59p.

1986b. Fly management in poultry production: cultural, biological, and chemical. Poultry Science, Champaign, 65:657-667.

Axtell, R. C. \& Arends, J. J. 1990. Ecology and management of arthropod pests of poultry. Annual Review of Entomology, Palo Alto, 35:101-126.

Axtell, R. C. \& StinNer, R. S. 1990. Computer simulation modeling of fly management. In: Rutz, D. A. \& Patterson, R. S. eds. Biocontrol of arthropods affecting livestock and poultry. Colorado, Boulder. p.265-291.

Bruno, T. V.; Guimarães, J. H. et al. 1993. Moscas sinantrópicas (Diptera) e seus predadores que se criam em esterco de aves poedeiras confinadas, no Estado de São Paulo, Brasil. Revista Brasileira de Entomologia, São Paulo, 37(3):577-590.

Colless, D. H. \& McAlpine, D. K. 1991. Diptera. In: Csiro. eds. The insects of Australia. 2. ed. Victoria, Melbourne University. v.2, p.717-787.

Curran, C. H. 1965. The families and genera of North American Diptera. 2. ed. New York, Woodhaven. 515p.

Fernandes, M. A.; Santos, M. A. S. \& Lomônaco, C. 1995. Ocorrência de artrópodes no esterco acumulado em uma granja de galinhas poedeiras. Anais da Sociedade Entomológica do Brasil, Porto Alegre, 24(3):649-654.

HaRwood, R. F. \& James, M. T. 1979. Entomology in human and animal health. 7. ed. New York, Macmillan. 548p.

Lomônaco, C. \& Prado, A. P. 1994. Estrutura comunitária e dinâmica populacional da fauna de dípteros e seus inimigos naturais em granjas avícolas. Anais da Sociedade Entomológica do Brasil, Londrina, 23(1):71-80.

Mariconi, F. A. M.; Guimarães, J. H. G. \& Berti Filho, E. 1999. A mosca doméstica e algumas outras moscas nocivas. Piracicaba, Fundação de Estudos Agrários Luiz de Queiroz. $135 \mathrm{p}$.

Odum, E. 1988. Ecologia. Rio de Janeiro, Guanabara Koogan. $434 p$.

Pfeiffer, D. G. \& Axtell, R. C. 1980. Coleoptera of poultry manure in caged-layer houses in North Carolina. Environmental Entomology, Lanham, 9:21-28.

Ricklefs, R. 1996. A economia da natureza. 3. ed. Rio de Janeiro, Guanabara Koogan. 470p.

Safrit, R. D. \& Axtell, R. C. 1984. Evaluations of sampling methods for darkling beetles (Alphitobius diaperinus) in the litter of turkey and broiler houses. Poultry Science, Champaign, 63(12):2368-2375.

Toвin, P. C. \& Pitts, C. W. 1999. Flotation method for extracting insects from poultry manure samples. Journal of Medical Entomology, Lanham, 36(1):121-123.

WALLNER, W. E. 1987. Factors affecting insect population dynamics: differences between outbreak and non-outbreak species. Annual Review of Entomology, Stanford, 32:317340.

Watson, D. W.; Waldron, J. K. \& Donald, A. R. 1994. Integrated management of flies in and around dairy and livestock barns. New York, Cornell University. 4p. 